\title{
New insight into rheology and flow properties of complex fluids with Doppler optical coherence tomography
}

\author{
Sanna Haavisto, Antti I. Koponen and Juha Salmela* \\ Fibres and Biobased Materials, Rheology and Process Flows, VTT Technical Research Centre of Finland, Jyväskylä, Finland
}

\section{Edited by:}

Jean-Michel Lavoie, Université de

Sherbrooke, Canada

\section{Reviewed by:}

Laurent Chrusciel, Université de Lorraine, France

Maria Graca Rasteiro, University of Coimbra, Portugal

*Correspondence:

Juha Salmela, VTT Technical

Research Centre of Finland, PO Box 1603, FIN-40401, Jyväskylä, Finland e-mail: juha.salmela@vtt.fi
Flow properties of complex fluids such as colloidal suspensions, polymer solutions, fiber suspensions and blood have a vital function in many technological applications and biological systems. Yet, the basic knowledge on their properties is inadequate for many practical purposes. One important reason for this has been the lack of effective experimental methods that would allow detailed study of the flow behavior of especially opaque multi-phase fluids. Optical Coherence Tomography (OCT) is an emerging technique capable of simultaneous measurement of the internal structure and motion of most opaque materials, with resolution in the micrometer scale and measurement frequency up to $100 \mathrm{kHz}$. This mini-review will examine the recent results on the use of Doppler-OCT in the context of flows and rheological properties of complex fluids outside biomedical field.

Keywords: optical coherence tomography, complex fluids, rheology, multi-component fluids

\section{INTRODUCTION}

Complex fluids are typically composed of several nonhomogeneously mixed components. These fluids are often homogeneous at macroscopic scales but disordered at microscopic scales and possess structures of mesoscopic length scales, which play a key role in determining the usually quite intricate properties of the fluid. Some examples of such length scales are the radii of micro emulsion drops, the dimensions of particles in liquid-particle suspension and bubble size in aerogels and foams. Synthetic and biopolymer solutions are one of the most important and widespread class of complex fluids but most multi-phase fluids have these properties. Although the importance of complex fluids is high and yet increasing, the basic knowledge on their properties is inadequate for many practical purposes. This is mainly because of two reasons. Firstly, the variation of structural, rheological, and optical properties of such fluids is extensive and makes it difficult to characterize and categorize them. Secondly, up to the very recent years, there has been lack of effective experimental methods that would allow detailed study of the flow dynamics and structure of especially opaque complex fluids (Gelbart and BenShaul, 1996; Barrat et al., 2005).

The rheology of simple fluids is rather straightforward and well understood. Flow behavior can be characterized with either a single temperature dependent coefficient of viscosity (Newtonian fluids) or with relatively simple relations between the stress and the strain rate (non-Newtonian fluids). Furthermore, these material properties can be accurately measured using conventional rheological methods. It is noteworthy, that the same methods are widely used also in studying the rheological properties of complex fluids. However, acquiring rheological data with complex fluids is not straightforward due to non-uniform and inconstant behavior such as apparent wall slip and wall depletion (Barnes, 1995), particle migration (Leighton and Acrivos, 1987) and shear banding (Olmsted, 2008) that can arise during the experiment.
Mechanisms underlying these complicated and poorly understood phenomena are related to the presence of the mesoscopic length scales and its consequences on boundary layer flow (Stickel and Powell, 2005). Fortunately, local rheological measurements can be used to overcome these problems.

The local rheological measurements are based on velocity profiling. This allows replacing an assumed velocity field by an actually measured profile in analyzing the data for rheological properties of the fluid. The local values of viscosity can be calculated as

$$
\mu(y)=\tau(y) / \dot{\gamma}(y),
$$

where $\dot{\gamma}(y)$ is the shear rate profile (local shear rate) derived from the measured velocity profile and $\tau(y)$ is the local shear stress obtained either from a pressure difference measurement (capillary or pipe flow geometry) or from a torque measurement (rotational rheometer). The local rheological measurements have captured some of the perturbative effects previously encountered in rheological experiments. For example, both structural probing and velocimetry techniques to detect shear-banded flows have been reviewed in (Manneville, 2008).

Complex fluids are typically highly scattering materials and thus optically turbid. Among the various traditional velocimetry techniques, only the Nuclear Magnetic Resonance Imaging (NMRI) and the Pulsed Ultrasound Doppler Velocimetry (PUDV) are capable of measuring the velocity field (and structure) of such (opaque and heterogeneous) fluids. Both PUDV and NMRI have been tested for several complex fluid and multiphase systems in tube flow and Couette geometry (Seymour et al., 1993, 1995; Britton and Callaghan, 1997; Raynaud et al., 2002; Ovarlez et al., 2011). Especially with tube flows the velocimetry concept is well established and has been implemented as in-line rheometers into industrial processes. These methods have provided novel means for process monitoring and quality control 
(Arola et al., 1997; Wunderlich and Brunn, 1999; Wiklund and Stading, 2008). However, due to the limited spatial resolution of 40-50 $\mu \mathrm{m}$ (Manneville, 2008) and with PUDV disturbances caused by the wall-fluid interface (Messer and Aidun, 2009), NMRI and PUDV are hardly accurate enough to resolve the flow profile in small flow geometries or in the immediate vicinity of solid boundaries, where e.g., apparent slip can occur.

Optical coherence tomography (OCT) has been developed rapidly in the past years. The use of OCT has been extended from its original application area, biomedicine, to many other fields. A review of OCT applications outside biomedical field (Stifter, 2007) presents several application cases from dimensional metrology and materials research to data storage and security applications. OCT has been introduced also for velocity profiling (Chen et al., 1997) but somewhat surprisingly, the potential of OCT in rheological applications has only very recently been realized (Harvey and Waigh, 2011; Lauri et al., 2011).

In this article we review some of the recent developments and applications of OCT outside of medical fields. We focus our review to the use of OCT to measure flow properties and rheology of complex fluids. At first, a very brief summary of the origin and basic principle of OCT with emphasis on velocity measurement (Doppler-OCT) is given. Finally, some recent promising studies are presented.

\section{PRINCIPLE OF OPTICAL COHERENCE TOMOGRAPHY ORIGIN AND METHODS}

OCT was introduced in the early nineties for non-invasive depthresolved imaging with micrometer resolution. Penetration depth depends greatly on optical properties of the material and used OCT principle. Penetration depth varies from micrometers to millimeters. The principle of OCT imaging is analogous to ultrasound, except that it utilizes light instead of acoustic waves, thereby achieving image resolutions of one to two orders of magnitude higher than standard ultrasound (Fujimoto, 2003). The functional principle of OCT is based on interpretation of the interference patterns of white or low-coherence light emitted to the sample. The interference pattern contains simultaneous information of the location, scattering index (density) and the travelling speed of the scattering particles (Stifter, 2007). In practice, OCT imaging is realized using a Michelson interferometer with a low-coherence-length light source, such as super luminescence diodes or solid state lasers (Fujimoto, 2003).

There are two main OCT methods that are classified based on the interferometric technique. In time domain OCT (TDOCT) the measurement volume is moved through the sample by changing the position of a reference mirror and the scattering interference is measured sequentially for all locations. The other option is frequency or spectral domain OCT (FD-OCT or SDOCT) where the whole imaging depth is captured instantaneously and no reference mirror movement is required. In this case the interference pattern is divided spectrally to different detectors and information from different measurement depths is analyzed simultaneously. For the physical fundamentals as well as comparative analysis on the OCT methods the reader is encouraged to refer to review articles or textbooks (e.g., Schmitt, 1999; Fercher et al., 2003; Brezinski, 2006; Drexler and Fujimoto, 2008).
In addition to cross-sectional tomography imaging OCT was soon adopted for measuring flow velocities. Coherence gating concept for measuring velocity profile of water flow in a duct was introduced in 1991 (Gusmeroli and Martinelli, 1991). The extension of OCT to measure flow velocities is called Doppler-OCT (D-OCT) or Optical Doppler Tomography (ODT). The Doppler frequency generated by moving objects is detected from the phase difference of two adjacent OCT measurements and it is proportional to the sample velocity. The velocity component parallel to the incident beam generates a Doppler shift to the signal and this that can be detected with a single component OCT system.

\section{VELOCIMETRY WITH DOPPLER OPTICAL COHERENCE TOMOGRAPHY (D-OCT)}

After the basis of D-OCT velocity profiling was established the method has been further developed for advanced velocimetry. D-OCT velocity profiling has been validated against laminar flow of microparticle suspensions in cylindrical and square conduits either in air or in conduits submerged in highly scattering media mimicking tissue (Wang et al., 1995, 1997; Chen et al., 1997). For fully developed pipe flow D-OCT velocity profile measurement can quantify the average flow but for this accurate determination of the Doppler angle is crucial (Drexler and Fujimoto, 2008). Thus, methods for accurate determination of the Doppler angle and velocities perpendicular to probe beam have been developed. These methods have improved possibilities to investigate flows in complex geometries or within biological systems where evaluation of the Doppler angle is unfeasible (see e.g., Piao and Zhu, 2003; Wu, 2004; Wang and Wang, 2010).

The most well-known target of D-OCT measurements has been the flow of blood in real and artificial blood vessels. Velocity mapping with D-OCT has been performed in different locations within sudden contraction (Proskurin et al., 2003, 2004) and Tand Y-shaped vessel junctions (Bonesi et al., 2007a,b, 2010). More recently, D-OCT has been used to investigate capillary-driven blood flow (Cito et al., 2012).

Besides the intense attention in measuring blood flow, the potential of OCT for many other fluid dynamical applications have been reported. Applications covering this topic have been reviewed thoroughly in Refs. (Brezinski, 2006; Stifter, 2007; Drexler and Fujimoto, 2008). We mention here only flows in micro-scale geometries that provide a natural and successful platform for D-OCT. Examples on microchannel flows can be found in Wang et al. (2004), Marks et al. (2004), Ahn et al. (2005), Lauri et al. (2008) and for micron-scale boundary layer flows in Kempe et al. (2007), Mujat et al. (2013), and Gao et al. (2013).

\section{APPLICATION OF D-OCT FOR MEASURING RHEOLOGY OF COMPLEX FLUIDS}

D-OCT is a very promising tool for studying rheology in capillaries (Lauri et al., 2011), in rotational rheometers (Harvey and Waigh, 2011) and in elongational rheology (Dufour et al., 2005). By combining a rotational rheometer with D-OCT simultaneous measurement of the real velocity profile across the rheometer gap together with the shear stress has been reported (Harvey and Waigh, 2011; Jaradat et al., 2012; Saarinen et al., 2014). When the size of the flow geometry exceeds the maximum measuring 
A
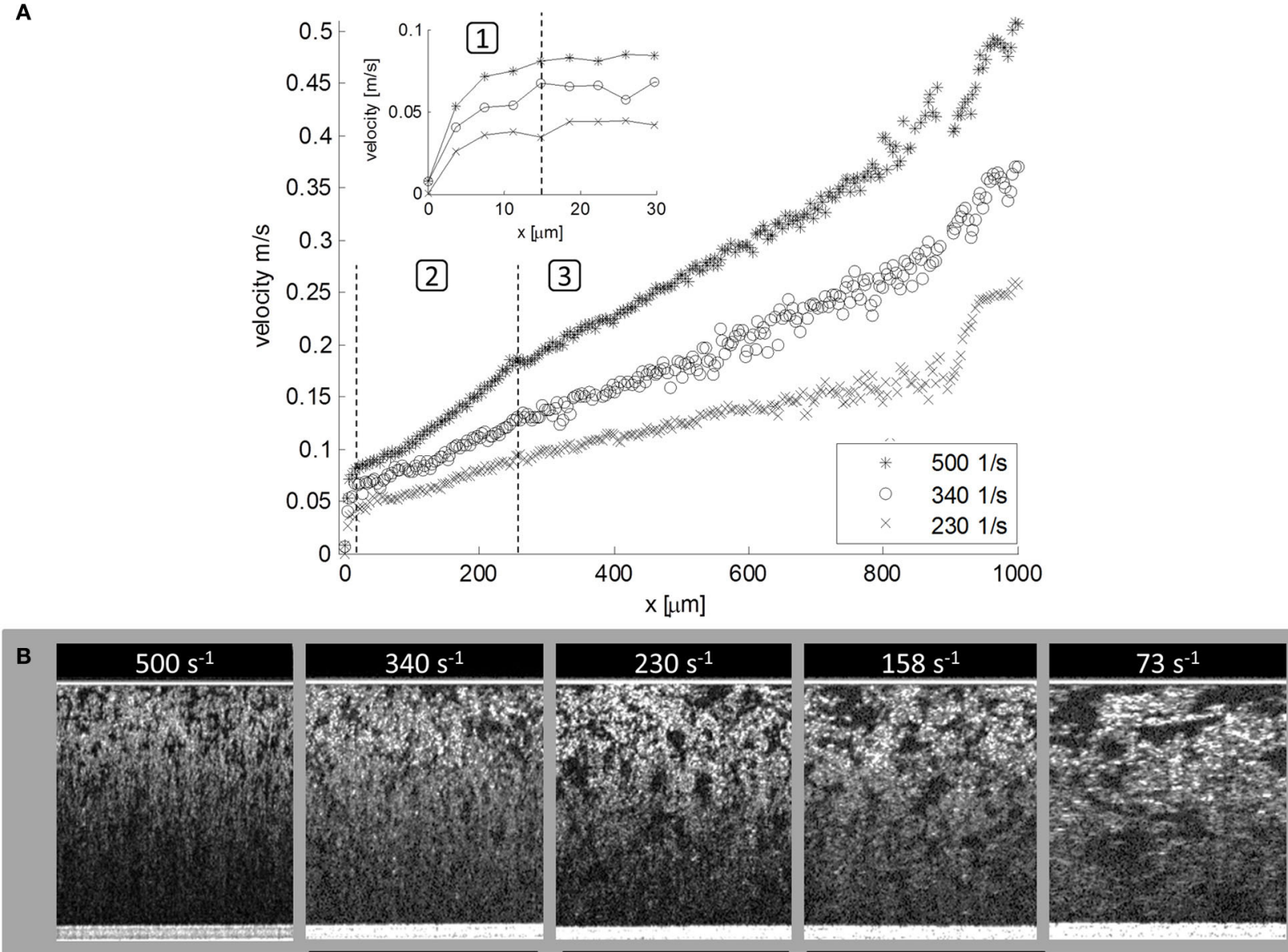

C
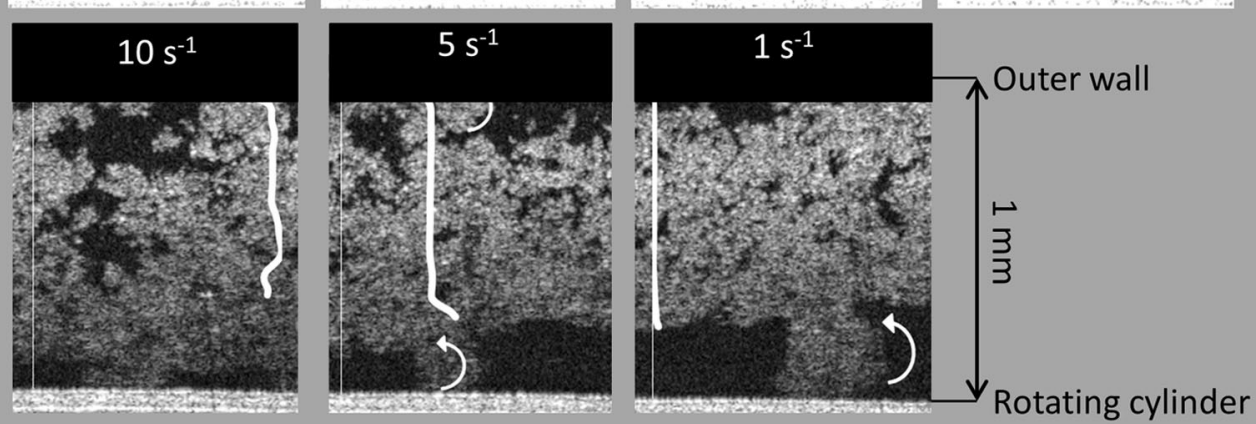

FIGURE 1 | OCT combined with a rotational rheometer equipped with smooth concentric cylinders geometry. (A) At high apparent shear rates the velocity profiles of $1 \%$ MFC have three regions: (1) Wall slip layer with thickness of $\sim 15 \mu \mathrm{m}$. (2) Wall boundary layer $(\sim 250 \mu \mathrm{m})$ where the slope of the velocity profile is higher than in the middle of the gap. (3) The outer layer, where the shear rates are $55-70 \%$ smaller than the apparent shear rate. Close to the moving boundary OCT profiles are distorted because the intensity of the scattering signal becomes too weak. (B) MFC suspension structure at moderate and high apparent shear rates. (C) Structure and velocity profiles for small apparent shear rates. From left to right: Complete slip on the stationary wall, slip and rolling flocs on both walls, and rolling flocs on the moving wall. Original figures are presented in Haavisto et al. (2014). distance of OCT the measurements can be complemented by measuring the outer flow velocity profile with e.g., PUDV or MRI techniques (Salmela et al., 2013). Combining such data with the structural information opens truly unprecedented research prospects for the study of rheology of opaque fluids.

One interesting material studied in this context is microfibrillated cellulose (MFC). MFC is produced from cellulose fibers using mechanical, chemical, or enzymatic disintegration methods. These fibrils have very high specific surface area, aspect ratio, strength, and flexibility. MFC fibers generally form an opaque strongly flocculated suspension with high viscosity and yield stress already at low mass concentrations (Pääkkö et al., 2007; Lavoine et al., 2012). These properties make the usage of most flow analysis methods very difficult. Rheology of MFC 


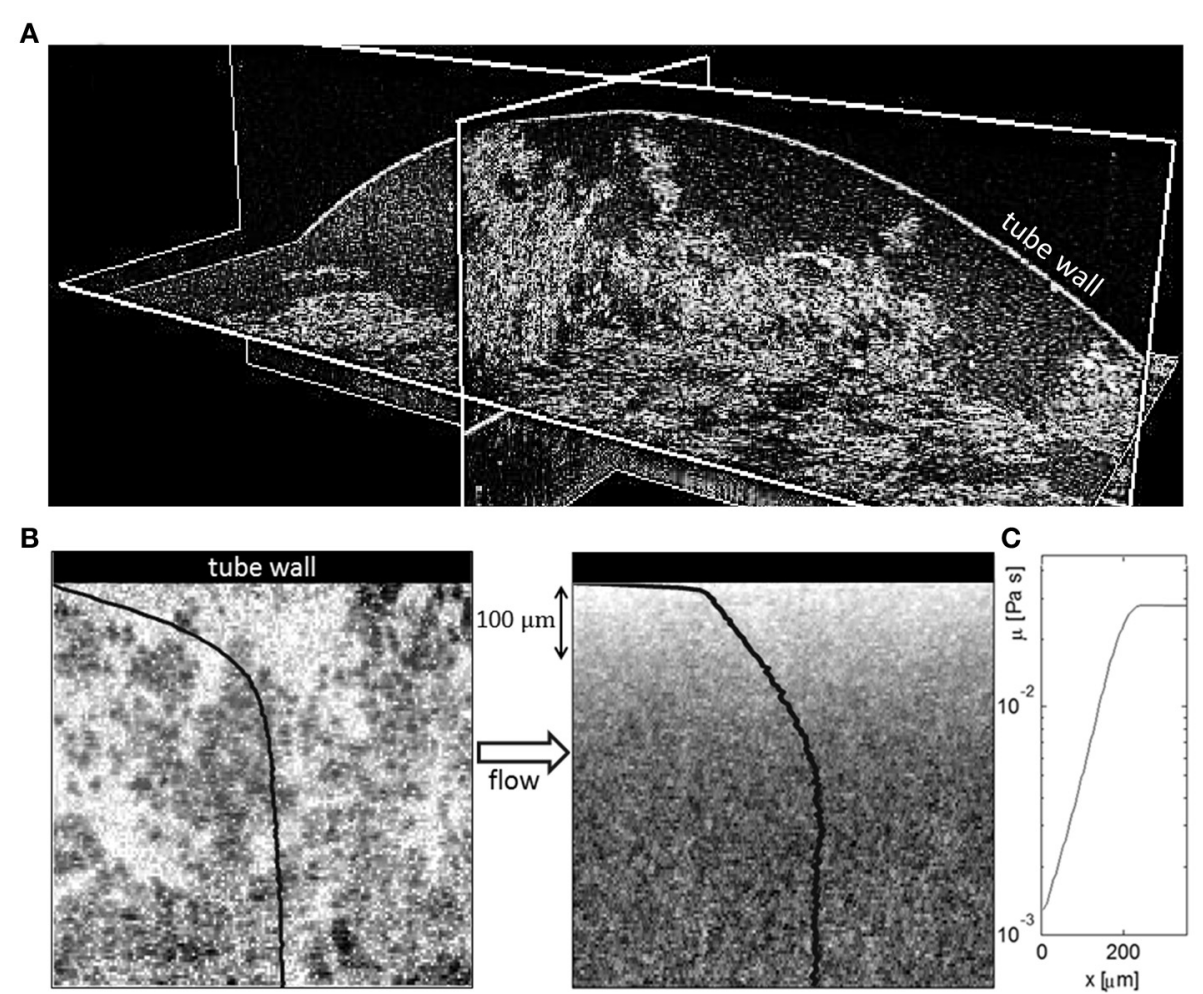

FIGURE 2 | (A) 3D OCT image of MFC suspension in a pipe with diameter of $8.6 \mathrm{~mm}$. Fiber-poor regions are clearly visible close to the pipe wall (curved surface). (B) 2D OCT image of flowing MFC (0.4\%) suspension near the tube wall. Shown are an instantaneous image (left) and an average of 200 independent images (right) acquired with SD-OCT. The gray-scale values in the image represent the local value of the optical back-scattering index, light color corresponding to low index value. The average velocity profile and the average scattering intensity i.e., effective concentration of the suspension are shown with black curves; the velocity profile on the left image and effective concentration on the right image. (C) The calculated viscosity profile close to the wall. Original figures are presented in Salmela et al. (2013) and Haavisto et al. (2014). suspensions has often been studied by interpreting shear viscosity from flow curve measurements (Agoda-Tandjawa et al., 2010; Iotti et al., 2011; Karppinen et al., 2012). Based on the flow curve MFC behaves like a strongly shear-thinning power law fluid $\left(\mu \sim \dot{\gamma}^{n}\right)$ with a Newtonian plateau between the low and high shear rate regions. A rheometer augmented with OCT reveals whether the flow curve actually represents true material properties of the suspension or if the flow curve is merely reflecting boundary layer phenomena like apparent wall slip (Haavisto et al., 2014; Saarinen et al., 2014). From the structure and velocity data the factors interfering with the rheological results are rather obvious (Figure 1). These results indicate that the MFC shear viscosity measured using rotational rheometer corresponds to suspension material properties only at high shear rates (after the Newtonian plateau). Even at the high shear rates three distinguished shear rate regions have been identified: Wall slip layer, wall layer and outer layer. In all the regions the velocity profiles differ from the ideal (assumed) one, which introduces some error to the apparent shear rate values.

Similarly as for MFC, non-ideal velocity profiles in rheometer gap have been demonstrated for margarine and for polymer solutions (Harvey and Waigh, 2011; Jaradat et al., 2012). Experiments performed with OCT rheometry captured shear-banding and wall slip in semi-dilute polyacrylamide (PAM) solutions when the molecular weight and the concentration of the polymer in the solution were high enough.

The mechanisms behind the non-ideal velocity profiles have been discussed by Salmela et al. (2013), Haavisto et al. (2014). OCT data has been analyzed separately for the structure information and for the local viscosity information. They report an almost fiber free layer very close to the pipe wall (Figure 2). In this layer the locally calculated viscosity is very close to that of water. With increasing distance the viscosity increases exponentially until it saturates to its bulk value approximately at $200 \mu \mathrm{m}$ from the wall. Contribution of this apparent slip layer may be as much as $95 \%$ to the total volumetric flow rate (Haavisto et al., 2014).

\section{CONCLUSION}

OCT activities are presently dominated by various biomedical applications, but we expect that the use of OCT will spread rapidly to numerous other fields. As the D-OCT method is capable of accurate high-resolution detection of simultaneous velocity and structure measurements it is exceptionally well suited in detailed study of the flow behavior and rheology of complex fluids in microfluidic devices or close to solid boundaries. Besides D-OCT there are many other OCT methods that are potentially very useful for rheological studies: Polarization sensitive OCT can be used for the analysis of particle and polymer orientation (Stifter 
et al., 2003), ultra-high resolution OCT (UH-OCT) will facilitate detailed understanding of the flow and structure down to submicron length-scales (Czajkowski et al., 2011), and dynamic light scattering OCT (DLS-OCT) can be utilized to obtain two or even three velocity components simultaneously (Huang and Choma, 2014). In the near future we expect to see more industrial applications where the OCT and PUDV (or MRI) techniques have been combined to study the real rheological properties of complex fluids. OCT will also give totally new insight in to the deformation of material in rheometer systems.

\section{ACKNOWLEDGMENTS}

We gratefully acknowledge valuable co-operation network of COST ACTION FP 1005 (Fibre Suspension Flow Modelling) and ERCOFTAG SIG 43 (Fibre Suspension Flows). Academy of Finland (project Rheological Properties of Complex Fluids) is gratefully acknowledged for supporting this work.

\section{REFERENCES}

Agoda-Tandjawa, G., Durand, S., Berot, S., Blassel, C., Gaillard, C., Garnier, C., et al. (2010). Rheological characterization of microfibrillated cellulose suspensions after freezing. Carbohydr. Polym. 80, 677-686. doi: 10.1016/j.carbpol.2009.11.045

Ahn, Y., Jung, W., Zhang, J., and Chen, Z. (2005). Investigation of laminar dispersion with optical coherence tomography and optical Doppler tomography. Opt. Exp. 13, 8164-8171. doi: 10.1364/OPEX.13.008164

Arola, D. F., Barrall, G. A., Powell, R. L., McCarthy, M. J., and McCarthy, K. L. (1997). Use of nuclear magnetic resonance imaging as a viscometer for process monitoring. Chem. Eng. Sci. 52, 2049-2057. doi: 10.1016/S00092509(97)00033-X

Barnes, H. A. (1995). A review of the slip (wall depletion) of polymer solutions, emulsions and particle suspensions in viscometers: its cause, character, and cure. J. Non Newtonian Fluid Mech. 56, 221-251. doi: 10.1016/03770257(94)01282-M

Barrat, J., Hansen, J., and Weeks, J. D. (2005). Basic concepts for simple and complex liquids. Phys. Today 58, 56. doi: 10.1063/1.1881906

Bonesi, M., Churmakov, D., and Meglinski, I. (2007b). Study of flow dynamics in complex vessels using Doppler optical coherence tomography. Meas. Technol. 18, 3279-3286. doi: 10.1088/0957-0233/18/11/003

Bonesi, M., Churmakov, D., Ritchie, L., and Meglinski, I. (2007a). Turbulence monitoring with Doppler Optical Coherence Tomography. Laser Phys. Lett. 4, 304-307. doi: 10.1002/lapl.200610098

Bonesi, M., Proskurin, S., and Meglinski, I. (2010). Imaging of subcutaneous blood vessels and flow velocity profiles by optical coherence tomography. Laser Phys. 20, 891-899. doi: 10.1134/S1054660X10070029

Brezinski, M. E. (2006). Optical Coherence Tomography: Principles and Applications. Academic Press.

Britton, M. M., and Callaghan, P. T. (1997). Two-phase shear band structures at uniform stress. Phys. Rev. Lett. 78, 4930-4933. doi: 10.1103/PhysRevLett.78.4930

Chen, Z., Milner, T. E., Dave, D., and Stuart Nelson, J. (1997). Optical Doppler tomographic imaging of fluid flow velocity in highly scattering media. Opt. Lett. 22, 64-66. doi: 10.1364/OL.22.000064

Cito, S., Ahn, Y., Pallares, J., Duarte, R. M., Chen, Z., Madou, M., et al. (2012). Visualization and measurement of capillary-driven blood flow using spectral domain optical coherence tomography. Microfluid. Nanofluidics 13, 227-237. doi: 10.1007/s10404-012-0950-6

Czajkowski, J., Myllylä, R., Jabbour, G., Fabritius, T., Ulanski, J., Marszalek, T., et al. (2011). Ultra-high resolution optical coherence tomography for encapsulation quality inspection. Appl. Phys. B 105, 649-657. doi: 10.1007/s00340-0114699-5

Drexler, W., and Fujimoto, J. G. (2008). Optical Coherence Tomography: Technology and Applications. Berlin, Heidelberg: Springer Berlin Heidelberg. doi: 10.1007/978-3-540-77550-8
Dufour, M., Lamouche, G., Detalle, V., Gauthier, B., and Sammut, P. (2005). Lowcoherence interferometry - an advanced technique for optical metrology in industry. Insight 47, 216-219. doi: 10.1784/insi.47.4.216.63149

Fercher, A. F., Drexler, W., Hitzenberger, C. K., and Lasser, T. (2003). Optical coherence tomography - principles and applications. Rep. Prog. Phys. 66, 239-303. doi: 10.1088/0034-4885/66/2/204

Fujimoto, J. G. (2003). Optical coherence tomography for ultrahigh resolution in vivo imaging. Nat. Biotechnol. 21, 1361-1367. doi: 10.1038/nbt892

Gao, Y., Haavisto, S., Tang, C., Salmela, J., and Li, W. (2013). Characterization of fluid dynamics in spacer-filled channels for membrane filtration using Doppler optical coherence tomography. J. Membr. Sci. 448, 198-208. doi: 10.1016/j.memsci.2013.08.011

Gelbart, W., and BenShaul, A. (1996). The "new" science of "complex fluids". J. Phys. Chem. 100, 13169-13189. doi: 10.1021/jp9606570

Gusmeroli, V., and Martinelli, M. (1991). Distributed laser Doppler velocimeter. Opt. Lett. 16, 1358-1360. doi: 10.1364/OL.16.001358

Haavisto, S., Salmela, J., Jäsberg, A., Saarinen, T., Sorvari, A., and Koponen, A. (2014). Rheological characterization of microfibrillated cellulose suspension using Optical Coherence Tomography. Tappi J. (accepted).

Harvey, M., and Waigh, T. A. (2011). Optical coherence tomography velocimetry in controlled shear flow. Phys. Rev. E 83, 31502. doi: 10.1103/PhysRevE.83. 031502

Huang, B. K., and Choma, M. A. (2014). Resolving directional ambiguity in dynamic light scattering-based transverse motion velocimetry in optical coherence tomography. Opt. Lett. 39, 521-524. doi: 10.1364/OL.39.000521

Iotti, M., Gregersen, Ø. W., Moe, S., and Lenes, M. (2011). Rheological studies of microfibrillar cellulose water dispersions. J. Polym. Environ. 19, 137-145. doi: 10.1007/s10924-010-0248-2

Jaradat, S., Harvey, M., and Waigh, T. A. (2012). Shear-banding in polyacrylamide solutions revealed via optical coherence tomography velocimetry. Soft Matt. 8, 11677-11686. doi: 10.1039/c2sm26395e

Karppinen, A., Saarinen, T., Salmela, J., Laukkanen, A., Nuopponen, M., and Seppälä, J. (2012). Flocculation of microfibrillated cellulose in shear flow. Cellulose 19, 1807-1819. doi: 10.1007/s10570-012-9766-5

Kempe, A., Schlamp, S., and Rösgen, T. (2007). Non-contact boundary layer profiler using low-coherence self-referencing velocimetry. Exp. Fluids 43, 453-461. doi: 10.1007/s00348-007-0320-4

Lauri, J., Bykov, A. V., and Myllylä, R. (2011). Determination of suspension viscosity from the flow velocity profile measured by Doppler Optical Coherence Tomography. Photonics Lett. Poland 3, 82-84. doi: 10.4302/plp. 2011.2.13

Lauri, J., Wang, M., Kinnunen, M., and Myllylä, R. (2008). "Measurement of microfluidic flow velocity profile with two Doppler Optical Coherence Tomography systems," in Progress in Biomedical Optics and Imaging Proceedings of SPIE, Vol. 6863 (San Jose, CA). doi: 10.1117/12.768500

Lavoine, N., Desloges, I., Dufresne, A., and Bras, J. (2012). Microfibrillated cellulose - its barrier properties and applications in cellulosic materials: a review. Carbohydr. Polym. 90, 735-764. doi: 10.1016/j.carbpol.2012.05.026

Leighton, D., and Acrivos, A. (1987). The shear-induced migration of particles in concentrated suspensions. J. Fluid Mech. 181, 415-439. doi: $10.1017 /$ S0022112087002155

Manneville, S. (2008). Recent experimental probes of shear banding. Rheologica Acta 47, 301-318. doi: 10.1007/s00397-007-0246-z

Marks, D. L., Parikh, D. S., Raskin, L., Boppart, S. A., and Hess, K. (2004). Structural and functional imaging of $3 \mathrm{~d}$ microfluidic mixers using optical coherence tomography. Proc. Natl. Acad. Sci. U.S.A. 101, 7516-7521. doi: 10.1073/pnas.0402433101

Messer, M., and Aidun, C. K. (2009). Main effects on the accuracy of PulsedUltrasound-Doppler-Velocimetry in the presence of rigid impermeable walls. Flow Meas. Instrum. 20, 85-94. doi: 10.1016/j.flowmeasinst.2008.11.002

Mujat, M., Ferguson, R. D., Iftimia, N., Hammer, D. X., Nedyalkov, I., Wosnik, M., et al. (2013). Optical coherence tomography-based microparticle image velocimetry. Opt. Lett. 38, 4558-4561. doi: 10.1364/OL. 38.004558

Olmsted, P. D. (2008). Perspectives on shear banding in complex fluids. Rheologica Acta 47, 283-300. doi: 10.1007/s00397-008-0260-9

Ovarlez, G., Mahaut, F., Bertrand, F., and Chateau, X. (2011). Flows and heterogeneities with a vane tool: Magnetic resonance imaging measurements. J. Rheol. (1978-present) 55, 197-223. doi: 10.1122/1.3526349 
Pääkkö, M., Ikkala, O., Lindström, T., Ankerfors, M., Kosonen, H., Nykänen, A., et al. (2007). Enzymatic hydrolysis combined with mechanical shearing and high-pressure homogenization for nanoscale cellulose fibrils and strong gels. Biomacromolecules 8, 1934-1941. doi: 10.1021/bm061215p

Piao, D., and Zhu, Q. (2003). Quantifying Doppler angle and mapping flow velocity by a combination of Doppler-shift and Doppler-bandwidth measurements in optical Doppler tomography. Appl. Opt. 42, 5158-5166. doi: 10.1364/AO.42.005158

Proskurin, S. G., He, Y., and Wang, R. K. (2004). Doppler optical coherence imaging of converging flow. Phys. Med. Biol. 49, 1265-1276. doi: 10.1088/00319155/48/17/311

Proskurin, S. G., Sokolova, I. A., and Wang, R. K. (2003). Imaging of non-parabolic velocity profiles in converging flow with optical coherence tomography. Phys. Med. Biol. 48, 2907-2918. doi: 10.1088/0031-9155/48/17/311

Raynaud, J., Moucheront, P., Baudez, J., Bertrand, F., Guilbaud, J., and Coussot, P. (2002). Direct determination by nuclear magnetic resonance of the thixotropic and yielding behavior of suspensions. J. Rheol. 46, 709-732. doi: $10.1122 / 1.1463420$

Saarinen, T., Haavisto, S., Sorvari, A., Salmela, J., and Seppälä, J. (2014). The effect of wall depletion on the rheology of microfibrillated cellulose water suspensions by Optical Coherence Tomography. Cellulose. doi: 10.1007/s10570-0140187-5. [Epub ahead of print].

Salmela, J., Haavisto, S., Koponen, A., Jäsberg, A., and Kataja, M. (2013). "Rheological characterization of micro-fibrillated cellulose fibre suspension using multi scale velocity profile measurements," in Proceeding of 15th Fundamental Research Symposium (Cambridge)

Schmitt, J. M. (1999). Optical Coherence Tomography (OCT): a review. IEEE J. Sel. Top. Quantum Electron. 5, 1205-1215. doi: 10.1109/2944.796348

Seymour, J. D., Maneval, J. E., McCarthy, K. L., McCarthy, M. J., and Powell, R. L. (1993). NMR velocity phase encoded measurements of fibrous suspensions. Phys. Fluids A Fluid Dyn. 5, 3010-3012. doi: 10.1063/1.858709

Seymour, J., Maneval, J., McCarthy, K., Powell, R., and McCarthy, M. (1995). Rheological characterization of fluids using NMR velocity spectrum measurements. J. Texture Stud. 26, 89-101. doi: 10.1111/j.1745-4603.1995. tb00786.x

Stickel, J., and Powell, R. (2005). Fluid mechanics and rheology of dense suspensions. Annu. Rev. Fluid Mech. 37, 129-149. doi: 10.1146/ annurev.fluid.36.050802.122132

Stifter, D. (2007). Beyond biomedicine: a review of alternative applications and developments for optical coherence tomography. Appl. Phys. B 88, 337-357. doi: 10.1007/s00340-007-2743-2

Stifter, D., Burgholzer, P., Höglinger, O., Götzinger, E., and Hitzenberger, C. K. (2003). Polarisation-sensitive optical coherence tomography for material char- acterisation and strain-field mapping. Appl. Phys. A Mater. Sci. Process. 76, 947-951. doi: 10.1007/s00339-002-2065-5

Wang, L., Xu, W., Bachman, M., Li, G. P., and Chen, Z. (2004). Phaseresolved optical Doppler tomography for imaging flow dynamics in microfluidic channels. Appl. Phys. Lett. 75, 1855-1857. doi: 10.1063/1. 1785854

Wang, X. J., Milner, T. E., and Nelson, J. S. (1995). Characterization of fluid flow velocity by optical Doppler tomography. Opt. Lett. 20, 1337-1339. doi: 10.1364/OL.20.001337

Wang, X., Milner, T., Chen, Z., and Nelson, J. (1997). Measurement of fluid-flowvelocity profile in turbid media by the use of optical Doppler tomography. Appl. Opt. 36, 144-149. doi: 10.1364/AO.36.000144

Wang, Y., and Wang, R. (2010). Autocorrelation optical coherence tomography for mapping transverse particle-flow velocity. Opt. Lett. 35, 3538-3540. doi: 10.1364/OL.35.003538

Wiklund, J., and Stading, M. (2008). Application of in-line ultrasound Doppler-based UVP-PD rheometry method to concentrated model and industrial suspensions. Flow Meas. Instrum. 19, 171-179. doi: 10.1016/j.flowmeasinst.2007.11.002

Wu, L. (2004). Simultaneous measurement of flow velocity and Doppler angle by the use of Doppler optical coherence tomography. Opt. Lasers Eng. 42, 303-313. doi: 10.1016/j.optlaseng.2003.08.005

Wunderlich, T., and Brunn, P. O. (1999). Ultrasound pulse Doppler method as a viscometer for process monitoring. Flow Meas. Instrum. 10, 201-205. doi: 10.1016/S0955-5986(99)00016-3

Conflict of Interest Statement: The authors declare that the research was conducted in the absence of any commercial or financial relationships that could be construed as a potential conflict of interest.

Received: 03 March 2014; paper pending published: 07 April 2014; accepted: 28 April 2014; published online: 19 May 2014.

Citation: Haavisto S, Koponen AI and Salmela J (2014) New insight into rheology and flow properties of complex fluids with Doppler optical coherence tomography. Front. Chem. 2:27. doi: 10.3389/fchem.2014.00027

This article was submitted to Chemical Engineering, a section of the journal Frontiers in Chemistry.

Copyright (c) 2014 Haavisto, Koponen and Salmela. This is an open-access article distributed under the terms of the Creative Commons Attribution License (CC BY). The use, distribution or reproduction in other forums is permitted, provided the original author(s) or licensor are credited and that the original publication in this journal is cited, in accordance with accepted academic practice. No use, distribution or reproduction is permitted which does not comply with these terms. 